\title{
Response surface methodology, modeling to improve mercury removal from aqueous solutions using L-cysteine functionalized multi-walled carbon nanotubes
}

\author{
Tabarinia H. ${ }^{1}$, Zazouli M.A. ${ }^{2,}{ }^{*}$, Yousefi Z. ${ }^{2}$, Kalankesh L.R. ${ }^{2}$ and Charati J.Y. ${ }^{3}$ \\ ${ }^{1}$ Student Research Committee, Mazandaran University of Medical Sciences, Sari, Iran \\ ${ }^{2}$ Department of Environmental Health Engineering, Faculty of Health, Mazandaran University of Medical Sciences, Sari, Iran \\ ${ }^{3}$ Department of Biostatics, Faculty of Health, Mazandaran University of Medical Sciences, Sari, Iran \\ Received: 03/04/2016, Accepted: 10/12/2018, Available online: 04/01/2019 \\ *to whom all correspondence should be addressed: e-mail: mzazouli@mazums.ac.ir \\ https://doi.org/10.30955/gnj.001984
}

\section{Abstract}

The aim of this study was to evaluate the mercury removal from aqueous solutions by using L-cysteine functionalized Multi-walled Carbon Nanotubes. The effect of $\mathrm{pH}$, adsorbent dose, contact time and mercury concentration in removal efficiency was evaluated. Multi -walled carbon nanotubes were functionalized with L-cysteine. The Response Surface Methodology (RSM) was used to find the optimum process parameters. The results showed that an increase in contact time, $\mathrm{pH}$ and adsorbent dosage resulted in an increase of the adsorption rate. However, removal efficiency decreases by increasing mercury concentration. The highest and lowest removal efficiencies of mercury were $89 \%$ and $17 \%$, respectively. The maximum adsorption rate was occurring at $120 \mathrm{~min}$. It is concluded that L-cysteine functionalized multi-walled carbon nanotubes is an effective adsorbent for removal from aqueous solutions.

Keywords: Carbon nanotubes, L-cysteine, mercury, absorption.

\section{Introduction}

Contaminating of the water sources with heavy metal is one of the most challenging environmental issues. Heavy metals are normally found in small amounts in natural waters, soils and rocks with high concentrations in industrial wastewater (Boparai et al., 2013). Industrial and agricultural activities are the major sources of the heavy metal which are releasing into the environment and consequently entering the human food chain (Zazouli et al., 2014b; Chen et al., 1999; Malakootian et al., 2013). Meanwhile, mercury is known as a toxic and stable metal in the environment. Several epidemiological studies have demonstrated that mercury not only cause to irreversible neurological damages, but also can cause diseases such as Alzheimer, Mina Mata, tremors Paresthesia, and loss of vision, hearing, smell and taste, memory loss (Girginova et al., 2010; Zhu et al., 2009). Mercury with metallic odorless and white silver color is the only metal that is liquid at room temperature. Mercury is widely used in various industries such as the paper industry, petrochemical industry, cosmetics, pharmaceutical and also agriculture (Zhu et al., 2009). According World Health Organization (WHO) suggestion $0.001 \mathrm{mg} \mathrm{L}^{-1}$ Maximum Concentration Level (MCL) mercury in drinking water. In addition, it was determined for natural mineral water, bottle drinking water and tap drinking water 0.001, 0.001 and 0.006 $\mathrm{mg} \mathrm{L} \mathrm{L}^{-1}$, respectively, by Institute of Standards and Industrial Research of Iran (Gibičar et al., 2009). Also, maximum mercury concentration in wastewater effluent was stated $10 \mu \mathrm{g} \mathrm{L}^{-1}$ by USEPA (Zazouli et al., 2014b; Chen et al., 1999). Existing used techniques remove mercury, such as chemical precipitation, oxidation and reduction process, electric flotation, ion exchange, reverse osmosis, evaporation, biological processes due to low efficiency are under question. Meanwhile, the use of adsorption process known as a cost-effective process (Maleki et al., 2011; Zazouli et al., 2013). The various substances have been studied as an adsorbent such as fruit shell of Terminalia Catappa (Inbaraj and Sulochana, 2006), sawdust (Šćiban et al., 2006), biomass of the marine macroalga Cytoseira Baccata (Herrero et al., 2005), mesocraps of orange and sour orange (Zazouli et al., 2014a), activated carbon from furfural (Yardim et al., 2003) and, biomass materials and waste tiers (Skodras et al., 2007). Least amount of adsorbent and high removal efficiency are two important factors in selecting adsorbent so, carbon nanotubes are the most suitable material (Zazouli et al., 2013). Carbon nanotubes (CNTs) are composed of different shapes and sizes graphite which are divided into single-walled (SWCNTs) and multi-walled carbon nanotubes (MWCNTs) based on the number of layers of carbon atoms in their walls (Ren et al., 2011). The chemical interaction between the metal ions and functional groups of CNTs level is the most important adsorption mechanism (Shamspur and Mostafavi, 2009). Modification surface properties of nanotubes can be 
reduces emissions adsorption sites by adding functional groups (Ganjkhanlou et al., 2014; Mansouri et al., 2016). That is reported that adding functional groups help to improve metal ions adsorption and recovery of CNTs dispersion in water (Ren et al., 2011). The aim of this study is to evaluate the mercury removal from aqueous solutions by using L-cysteine functionalized Multi-walled Carbon Nanotubes (L-MWCNTs) and, investigate the optimization of operation parameters such as $\mathrm{pH}$, adsorbent dose, contact time and mercury concentration in removal efficiency using Response surface methodology(RSM).

\section{Materials and methods}

\subsection{Chemical and reagent}

Multi-walled carbon nanotubes (MWCNT, O.D. $\times$ L.D. 7-15 $\mathrm{nm} \times 0.5-10 \mu \mathrm{m}, \leq>90 \%$ purity) and, L-cysteine were purchased from Sigma-Aldrich Co. Analytical standard of Mercury $\left(\mathrm{HgCl}_{2}\right)$ was purchased from Merck Co., Germany. Stock solutions (1000 mg/L) were prepared and serial dilutions of the stock solutions were obtained. Acid nitric $(0.1 \mathrm{~N})$ and $\mathrm{NaOH}(0.1 \mathrm{~N})$ for adjusting $\mathrm{pH}$ of the solution was purchased from Merck Co.

\subsection{Preparation of L-MWCNTS}

MWCNT functionalization was performed by adding $5 \mathrm{~g}$ of carbon nanotube in $50 \mathrm{ml}$ sulfuric (v/v 3:1). Then, nitric acid was dispersed in the solution and was suspended with sonication in an hour. The produced container was placed in a hot water bath at $50{ }^{\circ} \mathrm{C}$ and mixed with a magnetic stirrer for 8 hours and was cooled to room temperature. The oxidized nanotubes were isolated with 12,000 centrifuges and were rinsed repeatedly with Double-distilled water. Finally was dried under vacuum. Then oxidized nanotubes were dispersed in thionyl chloride at $60{ }^{\circ} \mathrm{C}$ degrees during 24 hours. In the next step, a solution of Triethylamine and Dimethylformamide solution was prepared from cysteine methyl ester salt. The nanotube prepared in the previous step was added to the environment. After 24 hours contact time at $55^{\circ} \mathrm{C}$, the nanotubes were separated and washed in three stages with deionized water. Finally, Modified nanotubes were put in the oven at $105{ }^{\circ} \mathrm{C}$ (Liu et al., 2008). Surface morphology and surface functional groups of L-MWCNTs were determined by Scanning Electron Microscope (SEM, KYKY factory, China) and Fourier transform infrared spectroscopy (FTIR), respectively. Atomic adsorption spectrophotometer (WFX-210, RAYLEIGH model) instrument was used for determining Mercury.

\subsection{Experimental design}

Number of required experiments and also investigate the combined effect of the independent variables were estimated by using regression coefficients and Box-Behnken mode (Zazouli et al., 2013). The four independent variables were $\mathrm{pH}, \mathrm{MWCNTs}$ dose, initial $\mathrm{Hg}$ concentration, and contact time. The number of tests required for model was determined by $\mathrm{N}=2 \mathrm{~K}(\mathrm{~K}-1)+\mathrm{C} 0$ equation that the $\mathrm{N}=$ number of samples (tests), $\mathrm{K}=$ number of factors (variables) and $\mathrm{C}_{0}=$ the central point number. A total of 27 experiments were introduced. Table 1 shows the ranges and levels of the independent variables. The low, middle, and high levels of each variable were designated as $-1,0$, and +1 respectively (Zazouli et al., 2013). A full quadratic model (FQM) was used to evaluate the obtained data. Optimizations were performed using Minitab 16 software. The experiments were conducted randomly to prevent systematic error. The coefficients of the full quadratic model were used to determine the $\mathrm{Hg}$ removal rate as an independent variable. The data were analyzed using the analysis of variance (ANOVA) and multiple regressions. The amount ( $p \leq 0.05$ ) was set at the level of significance. The ability of the final model was evaluated by numerical and graphical analysis using the Minitab 16 software. This statistical model gives the final form of the equation.

Table 1. The studied independent variables and their coded levels and actual values

\begin{tabular}{ccccc}
\hline Variable & $\mathbf{p H}$ & $\begin{array}{c}\text { Contact } \\
\text { time }\end{array}$ & $\begin{array}{c}\text { Hg } \\
\text { concentration }\end{array}$ & $\begin{array}{c}\text { L-MWXNTs } \\
\text { dose }\end{array}$ \\
\hline Unit & - & $\mathrm{min}$ & $\mathrm{mg} \mathrm{L}^{-1}$ & $\mathrm{mg} \mathrm{L}^{-1}$ \\
\hline Symbol & $\mathrm{X}_{1}$ & $\mathrm{X}_{2}$ & $\mathrm{X}_{3}$ & $\mathrm{X}_{4}$ \\
\hline+1 & 11 & 120 & 10 & 100 \\
\hline 0 & 7.5 & 65 & 5.05 & 55 \\
\hline-1 & 4 & 10 & 0.1 & 10 \\
\hline
\end{tabular}

\subsection{Batch experiments}

All the adsorption experimental setups was carried out according according Standard Methods for the Examination of Water and Wastewater guidelines (A.P.H. Association and A.W.W. Association, 1989). Removal efficiency of $\mathrm{Hg}$ was evaluated in different variables such as initial mercury concentration $\left(10,5.05\right.$ and $\left.0.1 \mathrm{mg} \mathrm{L}^{-1}\right)$; $\mathrm{pH}(4,7.5,11)$; contact time $(10,65$ and $120 \mathrm{~min})$; and MWCNTs dosage $(10,55$ and $100 \mathrm{~g})$. At the end of each step, the supernatant liquids were filtered using $0.2 \mu \mathrm{m}$ membrane filter to separate adsorbent. The purified samples were acidified by $\mathrm{HNO}_{3}$ to $\mathrm{pH}$ less than 2 . The acidify samples were stored in the $50 \mathrm{ml}$ glass containers in the refrigerator before analysis by Atomic adsorption spectrophotometer.

\section{Results and discussion}

\subsection{Characterization of L-MWCNTS}

SEM images of the MWCNT $_{S}(A)$ and, L-MWCNTs (B) was shown in following Figure 1 . Figure 2 shows the FTIR image of MWCNTs and L-MWCNTs. Obtained result confirms presence of functional and connective groups on carbon nanotubes. FTIR spectrums of MWCNTs and L-MWCNTs show the stretching vibration band of hydrogen bonded alcohol, $\mathrm{O}-\mathrm{H}$, around $3435 \mathrm{~cm}^{-1}$ that confirmed the presence of carbonyl on the surface of the nanotube. A vibration which is observed in the 3000 to 3200 is related to modify multi-walled carbon nanotubes in the form of the integration. In addition, $\mathrm{CH}_{2}$ - bond was correspond to vibrations in the $3435 \mathrm{~cm}^{-1}$. L-MWCNTs has the characteristic stretching of amide carbonyl $(\mathrm{C}=\mathrm{O})$ and amide II $\mathrm{v}(\mathrm{NH})$ stretching vibrations adsorption bands at 1637 and $1421 \mathrm{~cm}^{-1}$, respectively. 
The connection of a cysteine group to carbon nanotubes can be shown on the line appeared in the 1178 area which is related to the $\mathrm{C}-\mathrm{O}$ stretching vibration.

\subsection{Full quadratic model}

The correlations between the $\mathrm{Hg}$ removal efficiency and parameters for the L-MWCNTs are described by:

$$
Y=41+17.25(X 1)+13.9167(X 2)-10(X 3)+9(X 4)
$$$$
+12.7083(X 1)^{2}
$$

Where $\mathrm{Y}$ is the percentage removal of $\mathrm{Hg}(\%)$ and $\mathrm{X} 1, \mathrm{X} 2$, $\mathrm{X} 3$, and $\mathrm{X} 4$ represent the coded values for $\mathrm{pH}$, contact time, initial $\mathrm{Hg}$ concentration and L-MWCNTs dosage, respectively.

Table 2. Analysis of variance for the proposed model for $\mathrm{Hg}$ removal using L-MWXNTs

\begin{tabular}{cccccc}
\hline Source & DF & Sum of squares & Mean square & T & P-Value \\
\hline Regression & 14 & 9393.2 & 671.02 & 8.45 & $<0.00<$ \\
\hline Linear & 4 & 8066.8 & 2016.71 & 25.4 & $<0.001$ \\
\hline Square & 4 & 1157.4 & 289.34 & 3.64 & 0.036 \\
\hline Interaction & 6 & 170 & 28.33 & 0.36 & 0.892 \\
\hline Residual error & 12 & 952.8 & 79.4 & - & - \\
\hline Lack of fit & 10 & 950.8 & 95.08 & R-squared & 0.01 \\
\hline Pure Error & 2 & 2 & 1 & Adj R-squared & $90.79 \%$ \\
\hline Total & 26 & 10347 & - & & $80.05 \%$ \\
\hline
\end{tabular}

DF: Degrees of freedom

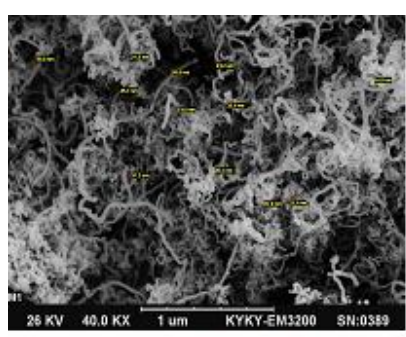

A) MWCNTs

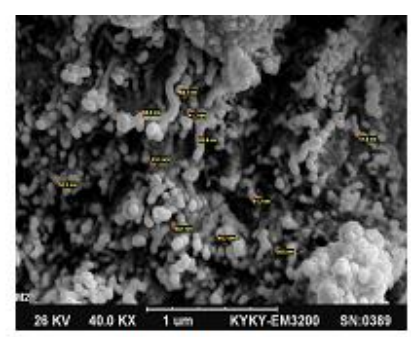

B) L-MWCNTs
Figure 1. SEM images of Multi-Walled Carbon Nanotubes and MWCNTs (A) and, L-MWCNTs (B). Magnification: 40,000x and Accelerating Voltage: $30-26000 \mathrm{~V}$

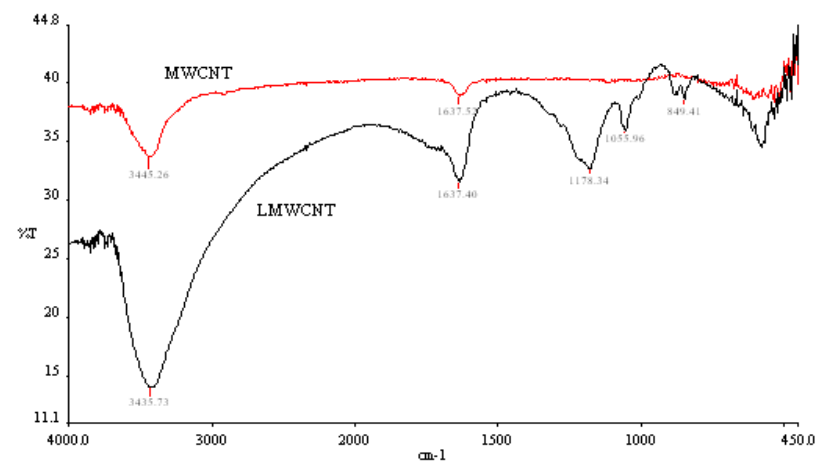

Figure 2. FTIR spectra of MWCNTs and L-MWCNTs

\subsubsection{Model validation}

To gain a suitable model, the results were summarized in a common ANOVA (Table 2). To confirm the goodness of fit between the model and the experimental results, regression coefficient (R-squared) analysis was applied. The adjusted R-squared values close to 1.0 indicate that the regression line fits the experimental data well (Asl et al., 2012). This equation can be used to predict the results at specific points with accuracies of $80.05 \%$. This model could also be used to predict the percentage of $\mathrm{Hg}$ removal at other adsorbents and times. The adjusted R-squared value is a modification of R-squared that adjusts for the number of explanatory terms in a model. Unlike R-squared, the adjusted R-squared value increases only if the new term improves the model more than would be expected by chance. The adjusted $\mathrm{R}$-squared valued can be negative and will always be less than or equal to R-squared. The adjusted R-squared value is more suitable for comparing models with different numbers of parameters (Asl et al., 2012). The adjusted $\mathrm{R}$-squared value is 0.8005 . Based on ANOVA analysis, experiment data in Table 2 show that the model statistically follows a linear pattern.

\subsubsection{Verification of the response surface model}

A scatter plot of results against predicted values from the model shows that the model is acceptable. The summary of variance analysis results for the L-MWCNTs are shown in Figure 3.

\subsection{Effect of $\mathrm{pH}$}

Figures 4-6 shows the interactions between the independent variables and the dependent variable for the mercury removal by using L-MWCNTs. Figure 4 reveals that the removal efficiency increases with increasing adsorbent dosage and increasing $\mathrm{pH}$. Also, Figure 6 shows that removal efficiency of mercury increases with increasing $\mathrm{pH}$ and contact time. In investigating of $\mathrm{pH}$ (4 to 11) with increasing contact time, the amount of mercury adsorption increased from 2.76 to $8.18 \mathrm{mg} \mathrm{g}^{-1}$ and also, removal efficiency increased $30 \%$ to $89 \%$. The maximum removal efficacy (89\%) was observed at $\mathrm{pH}=11$ with $5.05 \mathrm{mg} \mathrm{L}^{-1}$ initial mercury concentration, 120 minutes contact time and $55 \mathrm{mg}$ adsorbent dosage. In addition, in this condition maximum adsorption capacity of MWCNTs was $32.9 \mathrm{mg} \mathrm{g}^{-1}$. It will be demonstrated that even at low $\mathrm{pH}=4$ and 120 minutes contact time the removal efficiency can also be higher (70\%). Several researchers have reported that the adsorption capacity of adsorbents rises with an increase in $\mathrm{pH}$ value (Tajar et al., 2009; Wang et al., 2010). Zazouli et al. reported that the adsorption rate of mercury from aqueous solutions in using a single-walled carbon nanotubes functionalized by L-cysteine increased by increasing the $\mathrm{pH}$ and the concentration of carbon 
nanotubes (Anbia and Mohammadi, 2009). Also the result shows same resalt in investigating removal cadmium by single-walled and multi-walled carbon nanotubes functionalized by L-cysteine (Tajar et al., 2009; Wang and Xing, 2004). Decrease adsorption efficiency at $\mathrm{pH}<6.0$ is due to electrostatic repulsion between the surface of LMWCNTs, and the cationic mercury ions and the high concentration of $\mathrm{H}^{+}$in the solution (Tajar et al., 2009; Wang and Xing, 2004). On the other hand, number of the positively charged sites on the adsorbent surface decrease and the number of negatively charged sites increase by an increase in initial $\mathrm{pH}$ of the solution (Wang et al., 2010). Vukovic et al. have reported that maximum adsorption rate for multi-walled carbon nanotubes oxidized occurred at $\mathrm{pH}=6-10$ (Anbia and Mohammadi, 2009).
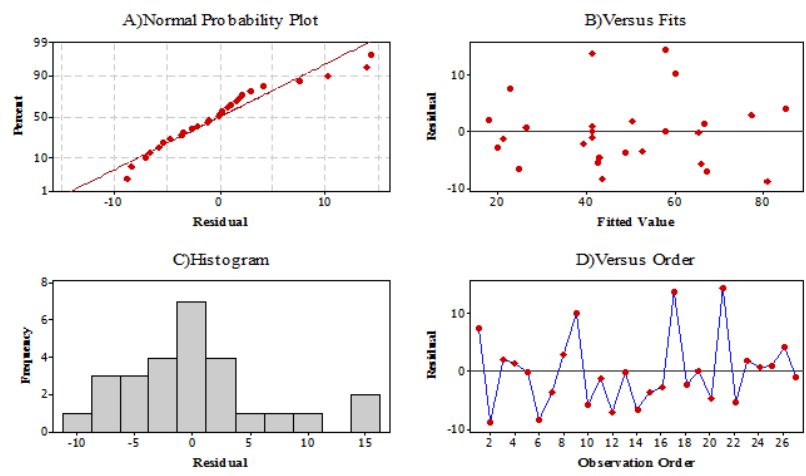

Figure 3. Residual plots for Hg removal via the L-MWCNTs

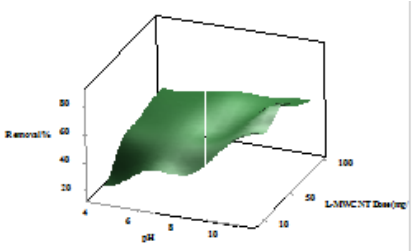

(A)

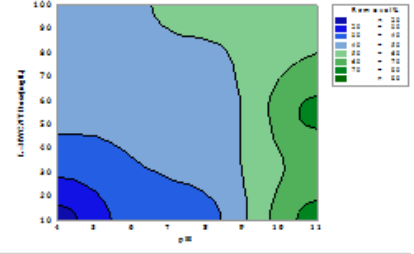

(B)
Figure 4. Response (A) surface plot and (B) contour plot of the mercury removal efficiency (\%) as a function of $\mathrm{pH}$ and adsorbent dosage

\subsection{Effect of L-MWCNT dosage}

Mercury removal efficiency as a function of $\mathrm{pH}$, adsorbent dosage and contact time, was illustrated in Figures 4 and 5. As obtained results mercury ions removal efficiency remarkably increased from 17 to $89 \%$ with an increase in L-MWCNTs dosage from 10 to $100 \mathrm{mg}$. Increase of adsorbent dosage resulted in greater availability of exchangeable sites for mercury ions (AlAnber and Matouq, 2008). This result is same with the other researches results (Zazouli et al., 2013, 2014b; Taghavi et al., 2015). Maleki et al. achieved to $90 \%$ removal eeficiency by increasing dosage until $5 \mathrm{~g}$ (Maleki et al., 2011). The absorbent structure can affect the behavior of adsorption. Also, by increasing the weight of absorbent, the mercury percentage will increase and the amount of mercury ions adsorbed per unit weight of adsorbent would be reduced (Puanngam and Unob, 2008). In addition, that is reported that by increasing the weight of granular activated carbon, the mercury removal rate increases and the ratio of absorbed mercury ions per unit mass will decrease (Zhang et al., 2010). Kosa et al. have investigated heavy metal removal efficiency and have founded that by an increase in adsorbent mass from 0.05 to $0.25 \mathrm{~g}$, the removal efficiency of $\mathrm{Cd}^{+2}, \mathrm{Zn}^{+2}, \mathrm{~Pb}^{+2}$ and $\mathrm{Cu}^{+2}$ reached 0-39\%; 6.58-97.68\%; 19.23-97.34\% and 99.83-99.94\%, respectively (Kosa et al., 2012).

\subsection{Effect of contact time}

The mercury removal efficiency as a function of contact time and adsorbent dosage. Shows at Figure 5. The mercury adsorption per unit mass of L-MWCNTs increases from $10.1 \mathrm{mg} \mathrm{g}^{-1}$ at $10 \mathrm{~min}$ contact time to $24.8 \mathrm{mg} \mathrm{g}^{-1}$ at $120 \mathrm{~min}$ contact time. Thus, the removal efficiency increases when contact time and the carbon nanotubes dosage increase. The maximum adsorption rate is $32.9 \mathrm{mg} / \mathrm{g}$ at $65 \mathrm{~min}$ contact time, which the removal efficiency of mercury is $68 \%$. In the initial contact time, more active sites and more functional groups were available so, lead to more removal efficiency of heavy metal (Saif et al., 2012). Available active sites and functional groups decreased with the passage the time so effect on the adsorption rate. Similar phenomena have been reported in the previous literature (White et al., 2009; Xiong et al., 2011). The optimum contact time for removal of mercury ions depend on the nanotubes dosage, $\mathrm{pH}$ and initial mercury concentration. Other research showed that mercury adsorption per unit mass of single-walled carbon nanotubes increases from 1.92 to $3.03 \mathrm{mg} \mathrm{g}^{-1}$ and the removal efficiency increases from 38 to 60 percent (Zazouli et al., 2014b). In the studywhich have done by at Iran on the removal of Biphenyl $A$ by activated carbon and egg shell also reported same result (Zazouli et al., 2013). Evaluation removal of $\mathrm{Cd}^{2+}$ by the monocarps of orange and sour orange also have same result (Zazouli et al., 2014b). In the study which have done by Lu et al., they reported that more time is needed to reach equilibrium adsorption and to increase adsorption efficiency by increasing the concentration of absorbent, correspond to the present study (Liu et al., 2008; Puanngam and Unob, 2008).
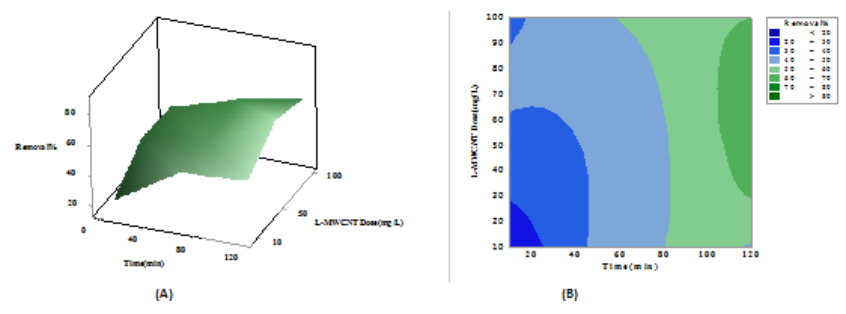

Figure 5. Response (a) surface plot and (b) contour plot of the mercury removal efficiency (\%) as a function of contact time and adsorbent dosage

\subsection{Effect of initial mercury concentration}

The results show that initial mercury concentration has opposite effect on the removal efficiency. The removal 
efficiency decreased from $72 \%$ at $0.1 \mathrm{mg} / \mathrm{l}$ of mercury concentration to $17 \%$ at $10 \mathrm{mg} / \mathrm{l}$ of mercury concentration (Figure 6). This phenomenon can be explain to saturation of active adsorption sites on L-MWCNT at the higher initial mercury concentration (Saif et al., 2012). In the other words, at lower mercury concentrations, the ratio of initial moles of mercury to the available adsorption sites is low, so, higher removal efficiency adsorption can be achieved (Boparai et al., 2013). With the increase in mercury concentration from 1.0 to 10 $\mathrm{mg} \mathrm{L}^{-1}$ at the contact time of 10 minutes, $\mathrm{pH}=7.5$ and adsorbent dosage of $55 \mathrm{mg}$, removal efficiency decreased from 45 to 27 percent; and in a contact time of 120 minutes, $\mathrm{pH}=7.5$ and adsorbent dosage of $55 \mathrm{mg}$, the removal efficiency decreased from 60 to 57 percent. In the study which have applied carbon nanotubes to removal of chromium adsorption efficiency decreased with increasing its initial concentration in the solution. The results were consistent with the present study (Hu et al., 2009). Other lecturel have also reported same result (Zazouli et al., 2013, 2014b).
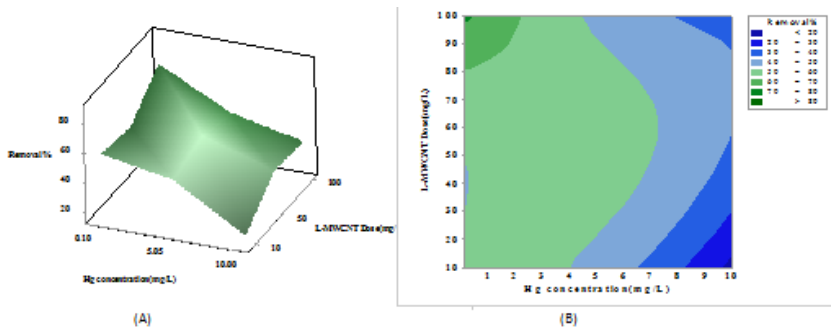

Figure 6. Response (A) surface plot and (B) contour plot of the mercury removal efficiency (\%) as a function of mercury concentration and carbon nanotubes dose

In compression efficiency of single-walled and multi-walled carbon nanotubes functionalized by L-cysteine in $\mathrm{Cd}^{+2}$ results showed that single-walled carbon nanotubes have higher efficiency than multi-walled carbon nanotubes. Also by increasing the $\mathrm{pH}$ and the concentration of carbon nanotubes, the removal efficiency increases (Zazouli et al., 2013). Also, another research reported that $\mathrm{Hg}^{+2}$ removal efficiency decreased by increasing of initial $\mathrm{Hg}$ concentration. In addition they mentioned that the adsorption rate increased by increasing of $\mathrm{pH}$, contact time and mass CNTs dose. And the maximum and the minimum adsorption rate were reported 95 and 22 percent, respectively (Zazouli et al., 2014b). However, the total amount of mercury adsorbed rapidly increased with an increase in the amount of initial mercury concentrations. High mercury concentration at the higher initial mercury concentrations, leaded a higher driving force for the mercury ions from the solution to the adsorbent (Gupta and Nayak, 2012; Tofighy and Mohammadi, 2011). Thus, more collisions occurred between mercury ions and active sites on the adsorbent. Therefore, the amount of adsorbed mercury ions on L-MWCNTs increased at higher initial cadmium concentration.

\section{Conclusion}

This research was performed to evaluate the efficiency of the multi-walled carbon nanotubes functionalized L-cysteine for mercury removal from aqueous solutions. The results reveal that the adsorption of mercury to L-MWCNTs is strongly dependent on initial pH, L-MWCNTs dosage, initial mercury concentration and contact time. With an increase in L-MWCNTs dosage, contact time and $\mathrm{pH}$ has a positive effect on the removal efficiency, which the removal efficiency of mercury ions increased. However, an increase in the initial concentration of mercury ions caused by an increase and decrease in the amount of mercury ions adsorbed per unit weight of the L-MWCNTs $\left(\mathrm{q}_{\mathrm{e}}\right)$ and the removal efficiency of mercury ions, respectively. Finally, the multi-walled carbon nanotubes functionalized L-cysteine is an effective adsorbent for metal removal from aqueous solutions especially mercury ions.

\section{Acknowledgments}

The authors would like to express their thanks to Department of Environmental Health Engineering,Health Sciences Research Center and the Research Deputy of Mazandaran University of Medical Sciences for the financial support of this study; and also the authors thank Dr. Behrouz Akbari-adergani and the laboratory staff of Food \& Drug Nanotechnology Research Center, Food \& Drug Organization, Ministry of Health for their contribution for preparing and functionalizing the MWCNTs. This project was performed using the research grant dedicated by Elites National Institute to Dr. Mohammad Ali Zazouli.

\section{References}

Al-Anber Z.A. and Matouq M.a.D. (2008), Batch adsorption of cadmium ions from aqueous solution by means of olive cake, Journal of Hazardous Materials, 151, 194-201.

Anbia M. and Mohammadi N. (2009), A nanoporous adsorbent for removal of furfural from aqueous solutions, Desalination, 249, 150-153.

A.P.H. Association and A.W.W. Association (1989), Standard Methods for the Examination of Water and Wastewater, American Public Health Association.

AsI S.K., Sadrnezaad S.K., Keyanpour-Rad M. and Üner D. (2012), Comparative photodecolorization of red dye by anatase, rutile (TiO_2), and wurtzite ( $\mathrm{ZnO})$ using response surface methodology, Turkish Journal of Chemistry, 36, 121-135.

Boparai H.K., Joseph M. and O'carroll D.M. (2013), Cadmium (Cd $2+)$ removal by nano zerovalent iron: surface analysis, effects of solution chemistry and surface complexation modeling, Environmental Science and Pollution Research, 20, 62106221.

Chen X., Feng X., Liu J., Fryxell G.E. and Gong M. (1999), Mercury separation and immobilization using self-assembled monolayers on mesoporous supports (SAMMS), Separation Science and Technology, 34, 1121-1132.

Ganjkhanlou Y., Bayandori Moghaddam A., Hosseini S., Nazari T., Gazmeh A. and Badraghi J. (2014), Application of image analysis in the characterization of electrospun nanofibers, Iranian Journal of Chemistry and Chemical Engineering (IJCCE), 33, 37-45.

Gibičar D., Horvat M., Logar M., Fajon V., Falnoga I., Ferrara R., Lanzillotta E., Ceccarini C., Mazzolai B. and Denby B. (2009), 
Human exposure to mercury in the vicinity of chlor-alkali plant, Environmental Research, 109, 355-367.

Girginova P.I., Daniel-Da-Silva A.L., Lopes C.B., Figueira P., Otero M., Amaral V.S., Pereira E. and Trindade T. (2010), Silica coated magnetite particles for magnetic removal of $\mathrm{Hg} 2+$ from water, Journal of Colloid and Interface Science, 345, 234-240.

Gupta V. and Nayak A. (2012), Cadmium removal and recovery from aqueous solutions by novel adsorbents prepared from orange peel and $\mathrm{Fe}_{2} \mathrm{O}_{3}$ nanoparticles, Chemical Engineering Journal, 180, 81-90.

Herrero R., Lodeiro P., Rey-Castro C., Vilariño T. and De Vicente M.E.S. (2005), Removal of inorganic mercury from aqueous solutions by biomass of the marine macroalga Cystoseira baccata, Water Research, 39, 3199-3210.

Hu J., Chen C., Zhu X. and Wang X. (2009), Removal of chromium from aqueous solution by using oxidized multiwalled carbon nanotubes, Journal of Hazardous Materials, 162, 1542-1550.

Inbaraj B.S. and Sulochana N. (2006), Mercury adsorption on a carbon sorbent derived from fruit shell of Terminalia catappa, Journal of Hazardous Materials, 133, 283-290.

Kosa S.A., Al-Zhrani G. and Salam M.A. (2012), Removal of heavy metals from aqueous solutions by multi-walled carbon nanotubes modified with 8-hydroxyquinoline, Chemical Engineering Journal, 181, 159-168.

Liu Y., Li Y. and Yan X.P. (2008), Preparation, characterization, and application of L-cysteine functionalized multiwalled carbon nanotubes as a selective sorbent for separation and preconcentration of heavy metals, Advanced Functional Materials, 18, 1536-1543.

Malakootian M., Ranandeh Kalankesh L. and Loloi M. (2013), Efficiency of hybrid nano particles of Tio2/Sio2 in removal of lead from paint industry effluents, Journal of Mazandaran University of Medical Sciences, 23, 244-254.

Maleki A., Mahvi A.H., Zazouli M.A., Izanloo H. and Barati A.H. (2011), Aqueous cadmium removal by adsorption on barley hull and barley hull ash, Asian Journal of Chemistry, 23, 1373.

Mansouri F., Kalankesh R. and Hasankhani H. (2016), The comparison of photo catalytic degradation of dissolved organic carbon (DOC) from water by UV/TiO2 in the presence and absence Iron ion. Global Nest Journal, 18, 392401.

Puanngam M. and Unob F. (2008), Preparation and use of chemically modified MCM-41 and silica gel as selective adsorbents for Hg (II) ions, Journal of Hazardous Materials, 154, 578-587.

Ren X., Chen C., Nagatsu M. and Wang X. (2011), Carbon nanotubes as adsorbents in environmental pollution management: a review, Chemical Engineering Journal, 170, 395-410.

Saif M.M.S., Kumar N.S. and Prasad M. (2012), Binding of cadmium to Strychnos potatorum seed proteins in aqueous solution: adsorption kinetics and relevance to water purification, Colloids and Surfaces B: Biointerfaces, 94, 73-79.

Šćiban M., Klašnja M. and Škrbić B. (2006), Modified softwood sawdust as adsorbent of heavy metal ions from water, Journal of Hazardous Materials, 136, 266-271.

Shamspur T. and Mostafavi A. (2009), Application of modified multiwalled carbon nanotubes as a sorbent for simultaneous separation and preconcentration trace amounts of $\mathrm{Au}$ (III) and $\mathrm{Mn}$ (II), Journal of Hazardous Materials, 168, 1548-1553.
Skodras G., Diamantopoulou I., Zabaniotou A., Stavropoulos G. and Sakellaropoulos G. (2007), Enhanced mercury adsorption in activated carbons from biomass materials and waste tires, Fuel Processing Technology, 88, 749-758.

Taghavi M., Zazouli M.A., Yousefi Z. and Akbari-Adergani B. (2015), Kinetic and isotherm modeling of Cd (II) adsorption by L-cysteine functionalized multi-walled carbon nanotubes as adsorbent, Environmental Monitoring and Assessment, 187, 682.

Tajar A.F., Kaghazchi T. and Soleimani M. (2009), Adsorption of cadmium from aqueous solutions on sulfurized activated carbon prepared from nut shells, Journal of Hazardous Materials, 165, 1159-1164.

Tofighy M.A. and Mohammadi T. (2011), Adsorption of divalent heavy metal ions from water using carbon nanotube sheets, Journal of Hazardous Materials, 185, 140-147.

Wang F.Y., Wang H. and Ma J.W. (2010), Adsorption of cadmium (II) ions from aqueous solution by a new low-cost adsorbent-Bamboo charcoal, Journal of Hazardous Materials, 177, 300-306.

Wang K. and Xing B. (2004), Mutual effects of cadmium and phosphate on their adsorption and desorption by goethite, Environmental Pollution, 127, 13-20.

White B.R., Stackhouse B.T. and Holcombe J.A. (2009), Magnetic $\mathrm{V}-\mathrm{Fe}_{2} \mathrm{O}_{3}$ nanoparticles coated with poly-l-cysteine for chelation of As (III), Cu (II), Cd (II), Ni (II), Pb (II) and Zn (II), Journal of Hazardous Materials, 161, 848-853.

Xiong L., Chen C., Chen Q. and Ni J. (2011), Adsorption of Pb (II) and Cd (II) from aqueous solutions using titanate nanotubes prepared via hydrothermal method, Journal of Hazardous Materials, 189, 741-748.

Yardim M.F., Budinova T., Ekinci E., Petrov N., Razvigorova M. and Minkova V. (2003), Removal of mercury (II) from aqueous solution by activated carbon obtained from furfural, Chemosphere, 52, 835-841.

Zazouli M.A., Ebrahimi P. and Ardebilian M.B. (2014a), Study of $\mathrm{Cd}$ (II) and $\mathrm{Cr}$ (VI) biosorption by mesocarps of orange and sour orange from aqueous solution, Environmental Engineering \& Management Journal (EEMJ), 13.

Zazouli M.A., Yousefi Z., Taghavi M., Akbari-Adergani B. and Yazdani Cherati J. (2013), Removing cadmium from aqueous environments using L-cysteine functionalized single-walled carbon nanotubes, Journal of Mazandaran University of Medical Sciences, 23, 37-47.

Zazouli M.A., Yousefi Z., Yazdani Cherati J., Tabarinia H., Tabarinia F. and Akbari Adergani B. (2014b), Evaluation of LCysteine functionalized single-walled carbon nanotubes on mercury removal from aqueous solutions, Journal of Mazandaran University of Medical Sciences, 24, 10-21.

Zhang Y., Li Q., Sun L., Tang R. and Zhai J. (2010), High efficient removal of mercury from aqueous solution by polyaniline/humic acid nanocomposite, Journal of Hazardous Materials, 175, 404-409.

Zhu J., Deng B., Yang J. and Gang D. (2009), Modifying activated carbon with hybrid ligands for enhancing aqueous mercury removal, Carbon, 47, 2014-2025. 